\title{
Evolución de la dactilogirosis (monogeneos) en branquias de alevinos de gamitana (Colossoma macropomum) en dos medios de crianza: acuático artificial y semi-natural
}

\author{
Evolution of dactilogiroses (monogeneans) in gills of tambaqui fry \\ (Colossoma macropomum) in two rearing aquatic media: artificial and semi- \\ natural \\ Juan Rondón E. ${ }^{1,4}$, César Villanueva C. ${ }^{1}$, César Gavidia $C^{2}$, \\ Víctor Puicón N. ${ }^{3}$
}

\section{Resumen}

\begin{abstract}
El objetivo del estudio fue determinar la evolución de la infestación por monogeneos de la familia Dactylogiridae en branquias de alevinos de gamitana (Colossoma macropomum) criados en jaulas en un medio acuático artificial y semi-natural. Se evaluaron 120 alevinos de 45 días de edad un peso promedio de $3.05 \pm 0.58 \mathrm{~g}, \mathrm{y}$ longitud total promedio de $5.23 \pm 0.51 \mathrm{~cm}$. Los peces fueron distribuidos en dos jaulas flotantes ( 60 alevinos por jaula), la primera ubicada en un estanque de tierra en una piscigranja con crianza semi-intensiva (medio artificial), y la segunda en un embalse (medio semi-natural). Ambos grupos recibieron similar régimen alimenticio. Los grados cualitativos de infestación considerados fueron leve, moderado y severo, según la cantidad de parásitos monogeneos encontrados en los cortes de branquias evaluados por microscopía directa. Los alevinos estuvieron libres de la infestación parasitaria al inicio del experimento, posteriormente se evaluaron 12 ejemplares por grupo a los 15, 30, 45, 60 y 90 días. A los 15 días, la infestación aumentó a grado leve en el medio semi-natural y continuó como negativo en el medio artificial; finalmente se tornaron a grado leve en ambos medios, hasta finalizar el experimento, sin haber diferencia significativa
\end{abstract}

\footnotetext{
${ }^{1}$ Estación del Centro de Investigación IVITA Pucallpa, Facultad de Medicina Veterinaria, Universidad Nacional Mayor de San Marcos, Ucayali, Perú

${ }^{2}$ Laboratorio de Medicina Veterinaria Preventiva, Facultad de Medicina Veterinaria, Universidad Nacional Mayor de San Marcos, Lima, Perú

${ }^{3}$ Escuela Profesional de Medicina Veterinaria, Facultad de Ciencias Agrarias, Universidad Nacional de San Martín - Tarapoto, Tarapoto, Perú

${ }^{4}$ E-mail:jrondone@unmsm.edu.pe
} 
entre grados de infestación en ambos grupos. Se concluye que ambos medios acuáticos no representan un riesgo para el desarrollo de infestaciones moderadas o severas por monogeneos en branquias que afecten las poblaciones de alevinos de gamitanas.

Palabras clave: monogeneos, branquias, gamitanas, alevinos, medios acuáticos, comunidades nativas

\section{Abstract}

The aim of this study was to determine the evolution of infestation by monogeneans of the Dactylogiridae family in gills of tambaqui fry (Colossoma macropomum) raised in cages in an artificial and semi-natural aquatic environment. In total, 120 45-day-old fingerlings were evaluated with an average weight of $3.05 \pm 0.58 \mathrm{~g}$, and an average total length of $5.23 \pm 0.51 \mathrm{~cm}$. The fish were distributed in two floating cages ( 60 fingerlings per cage), the first located in an earthen pond in a semi-intensive breeding farm (artificial environment), and the second in a reservoir (semi-natural environment). Both groups received a similar diet. The qualitative degrees of infestation considered were mild, moderate and severe, according to the number of monogeneans parasites found in the gill sections evaluated by direct microscopy. The fingerlings were free of the parasitic infestation at the beginning of the experiment, later 12 specimens were evaluated per group at 15, 30, 45, 60 and 90 days. At day 15, the infestation increased to a mild degree in the semi-natural environment and continued as negative in the artificial environment; later on they became mild in both media, until the end of the experiment, with no significant difference between degrees of infestation in both groups. It is concluded that both aquatic environments do not represent a risk for the development of moderate or severe infestations by monogeneans in gills that affect populations of tambaqui fry.

Key words: monogeneans, gills, tambaqui, fingerlings, aquatic environments, indigenous communities

\section{INTRODUCCIÓN}

La piscicultura es una actividad tradicional en la Amazonía Peruana, desarrollándose mayormente de manera extensiva y semi-intensiva con proyección a la crianza intensiva. En estos sistemas, la gamitana (Colossoma macropomum) una de las especies que ha tenido mayor crecimiento productivo en la Amazonía (Amanajás et al., 2018).

Las condiciones ambientales de las piscigranjas, como estación, temperatura y- salinidad son propicias para el desarrollo de las parasitosis, especialmente de monogeneos (Hoai, 2020). Entre las parasitosis más frecuentes en gamitanas se encuentra la dactilogirosis (Flores J y Flores R, 2003; Vargas et al., 2015). Entre las especies halladas se encuentran Anacanthorus spathulatus, Anacanthorus penilabiatus y Linguadactyloides brink-manni (Kritsky et al., 1979; Thatcher y Kritsky, 1983), así como Notozothecium janauachensis (BelmontJegú, 2004). Cabe indicar que la presencia de parásitos refleja la calidad sanitaria del ecosistema acuático y la contaminación ambiental (Sures et al., 2017). 
El índice de densidad parasitaria se relaciona con las lesiones halladas en branquias, superficie corporal y otras partes del cuerpo (Scholz y Kuchta, 2005; Fujimoto et al., 2019). Las lesiones más frecuentes son hiperplasia de lamelas primarias, necrosis, edema, displasia del epitelio respiratorio, ruptura de células pilar y telangiectasia; además, los monogeneos pueden actuar como endoparásitos a nivel gástrico, utilizando una ventosa oral anterior (Martins y Romero, 1996).

La crianza en jaulas dentro de embalses puede ser una alternativa para aminorar la sobrepesca, debido a que la densidad deja de ser un problema (Sierra y Maroso, 2019). El cultivo en jaulas ha crecido rápidamente, en parte debido a la mayor demanda mundial de productos acuáticos. Las jaulas y estanques ofrecen varias ventajas respecto a otros métodos de cultivo, puesto que se colocan en masas de agua ya existentes, lo que requiere inversiones de capital relativamente bajas y tecnología sencilla, gozan de gran popularidad entre los piscicultores y en los programas de desarrollo, dado que la crianza en jaulas no altera la tasa de crecimiento ni la ganancia de peso ante un aumento en las densidades de alevinos (Gomes et al., 2006). No obstante, el desarrollo de estos se asocia a la aparición de enfermedades parasitarias, por la mayor densidad de alevinos (Murray y Peeler 2005).

La calidad de agua de los estanques ha sido estudiada en función de sus parámetros químicos, no siendo un requerimiento especial para especies como el paiche (Arapaima gigas) (Pontes, 1977); sin embargo, en otras especies como el paco (Piaractus brachypomus) (Dinis, 2014), la temperatura se considera como uno de los factores más importantes para controlar la tasa de reproducción y supervivencia de monogeneos (Bakke et al., 2007; Winger et al., 2008).

En ambientes acuáticos naturales o semi-naturales, como el desarrollado en comunidades nativas, no se conoce el impacto de los agentes parasitarios frecuentes que podrían generar mermas en la población. Así mismo, no se dispone de información sobre la evolución de la parasitosis en branquias en estos sistemas acuáticos, teniendo en cuenta similares condiciones de densidad poblacional, edad y alimentación. Por lo tanto, el objetivo del presente estudio fue determinar la evolución de la infección por monogeneos en branquias de alevinos de gamitana (Colossoma macropomum) en medios de crianza acuático artificial y semi-natural.

\section{Materiales y Métodos}

\section{Lugar y Tiempo de Estudio}

El estudio se realizó en la Unidad de Producción de Peces Migratorios de la piscigranja de la Estación Experimental del Instituto Veterinario de Investigaciones Tropicales y de Altura (IVITA-Pucallpa), de la Facultad de Medicina Veterinaria, Universidad Nacional Mayor de San Marcos. La piscigranja se encuentra ubicada en el distrito de Campo Verde, provincia de Coronel Portillo, departamento de Ucayali, Perú.

La zona presenta un clima cálido húmedo, temperatura media de $26.7^{\circ} \mathrm{C}$, precipitación pluvial entre 79 y $642 \mathrm{~mm} / \mathrm{mes}$ y $85 \%$ humedad relativa media. Los peces fueron alojados en un estanque y en un embalse (espejo de agua ubicado a $150 \mathrm{~m}$ de distancia). El estudio se desarrolló entre marzo y julio de 2014.

\section{Peces y Fuentes de Agua}

Se recolectaron 144 alevinos de gamitana de 42 días de edad, con peso promedio de $3.05 \pm 0.58 \mathrm{~g}$ y una longitud total promedio de $5.23 \pm 0.51 \mathrm{~cm}$., de una piscigranja privada con un tipo de crianza semi-intensiva de gamitanas cercana a la piscigranja del IVITA. Se realizó el monitoreo previo de 30 alevinos provenientes de dicha piscigranja para determinar la frecuencia de 
la infestación por monogeneos Dactylogyrus en tejidos frescos de branquias por microscopía directa, encontrándose infestaciones leves en el 86.7\% (26/30) de los alevinos.

Los 144 alevinos fueron alojados en un tanque circular de policloruro de vinilo (PVC) del IVITA. Al día siguiente se evaluaron 12 alevinos al azar para determinar la frecuencia de parásitos en branquias y con los 132 peces restantes se realizó un tratamiento mediante inmersión en formalina diluida a (250 $\mathrm{mg} / \mathrm{L}$ ) por 30 minutos con abundante aireación, de acuerdo con el protocolo descrito por Conroy (2006). Luego fueron introducidos a otro tanque circular y fuente de agua. Al tercer día se evaluaron otros 12 alevinos tomados al azar para verificar la ausencia de parásitos postratamiento.

Los 120 alevinos de 45 días negativos a monogeneos en branquias fueron distribuidos equitativamente en dos jaulas flotantes, cada una de $4 \mathrm{~m}^{2}$ de área y $1.5 \mathrm{~m}$ de profundidad. Una de ellas ubicada en un estanque de tierra, preparado sanitariamente para la crianza de alevinos (medio artificial) mediante aplicación de abono y encalado, y la otra en un embalse (medio semi-natural). Ambos grupos recibieron un régimen similar de alimentación. Previo a la introducción de los peces a las dos fuentes de agua, se realizó un análisis fisicoquímico, microbiológico y parasitológico del agua. Dentro del diseño de experimentación, se sacrificaron 12 alevinos por medio de cultivo por periodo experimental

Para los análisis fisicoquímico y parasitológico del agua se usó el método SMEWW-APHA-AWWA-WEF Ed.22 2012, y para el análisis microbiológico se usó el método APHA/AWWA/WEF 2005 para determinar el Número más probable (MNP) de coliformes termotolerantes, Escherichia coli y Pseudomona aeruginosa en agua.

\section{Evaluación Microscópica}

Las evaluaciones de seguimiento mediante toma de muestras y evaluación microscópica se realizaron a los 15, 30, 45, 60 y 90 días. El sacrificio de los alevinos se realizó considerando los principios de bienestar animal y las leyes de protección animal vigentes. El procedimiento de anestesia se realizó mediante inmersión de los peces en una infusión de $3 \mathrm{~g} / 1$ de polvo de clavo de olor (Syzygium aromaticum) (fuente natural de Eugenol, principio activo con propiedades anestésicas). La inmersión se realizó durante 45 segundos para la anestesia y sacrificio posterior. La necropsia y extracción de las branquias en fresco se realizó según lo descrito por Eiras et al. (2006) y Conroy (2006).

La evaluación microscópica se realizó en tres campos al azar, ubicados en la base, parte media y ápice del filamento branquial, proveniente de la zona apical de la segunda capa branquial de ambos lados, lo que sumó un total de seis campos. Cada corte de la branquia fue preparada en láminas portaobjetos para la observación microscópica (10X) de trematodes monogeneos. Los grados de infección fueron considerados de acuerdo con ensayos previos realizados en alevinos y de acuerdo con lo propuesto por Klinger y Floyd (2002). La identificación de los parásitos monogeneos compatibles con Dactilogyrus fue de acuerdo con las características morfológicas y morfométricas establecidas por Cohen et al. (2013). El número de formas parasitarias compatibles con monogeneos en los cortes de branquias (dos cortes por ejemplar, uno del lado izquierdo y uno del derecho) fueron categorizados en grados leve (1-3), moderado (4-7) y severo ( $\geq 8$ ).

\section{Análisis de Datos}

Se detallaron los parámetros del análisis de las dos fuentes de agua y los grados de parasitosis en los alevinos de los dos medios 
de cultivo mediante estadística descriptiva. Asimismo, se determinó la diferencia de medias entre los grados de parasitosis en los dos medios de cultivo mediante el análisis de su intervalo de confianza al 95\%.

\section{Resultados}

El análisis de los parámetros fisicoquímicos y microbiológicos de las fuentes de agua se presentan en el Cuadro 1. Los parámetros, a excepción del hierro, se encuentran por debajo de los límites máximos permisibles indicados por las autoridades pertinentes, tales como la Organización Mundial de la Salud (OIE), y el Ministerio del Ambiente y el Ministerio de Salud del Perú.

Las medianas del número de monogeneos encontrados en los cortes de branquias en los 12 alevinos por periodo experimental se muestra en el Cuadro 2. Se evidencia que la infestación por monogeneos (principalmente Dactylogyrus spp) aumentó a leve en el medio semi-natural y continuó negativo en el medio artificial; sin embargo, posteriormente resultó negativo en el medio semi-natural y aumentó a leve en el medio artificial, terminando en grado leve en ambos medios. El índice del intervalo de confianza fue de 0.2921 $\leq \mu_{1}-\mu_{2} \leq 0.5078$. Por lo tanto, al no incluir al 0 , no existe diferencia significativa entre las medias de infestación parasitaria entre ambos grupos experimentales.

\section{Discusión}

Los monogeneos son parásitos comensales que se alimentan de la superficie de la piel, tejidos branquiales y otras zonas corporales (Fujimoto et al., 2019), pero su presencia esta correlacionada a factores como calidad de agua y densidad de alevinos, lo cual puede generar infecciones oportunistas (Aly et al., 2020). Es así que, en condiciones naturales, se ha reportado prevalencias de hasta $49.2 \%$ de monogeneos en Colosoma macropomum en el nordeste de Brasil (Fujimoto et al., 2019); 70\% de monogeneos en dos centros de cultivo de tilapia (Oreochromis spp) en Lima (Gonzales, 2012) y $98.4 \%$ en el estado de Portuguesa, Venezuela (Aragort et al., 1997).

La parasitosis por monogeneos, según las condiciones de cultivo, puede llegar a niveles patológicos (Scholz y Kuchta, 2005). Las altas densidades y la mala calidad del agua pueden exacerbar los cuadros, especialmente si se realiza una deficiente manipulación que ocasiona situaciones de estrés en los peces (Bowers et al., 2000; Collins et al., 2000). Cabe considerar que el desarrollo del sistema inmune incluye a la acción defensiva del tejido linfoide en branquias como primer mecanismo de defensa, Luo y Yang (2010) determinaron una correlación positiva entre la infestación por monogeneos y los peces de tamaño más pequeño, probablemente debido al menor desarrollo del sistema inmunológico de los peces jóvenes.

La crianza en jaulas colocadas en embalses y en estanques manejando similares condiciones de densidad y alimentación está influenciada por la calidad de agua, siendo así que, en jaulas colocadas en embalses, el espejo de agua es mayor por lo que habría un recambio de agua en el volumen de crianza de los peces, lo cual dificultaría el reinicio del ciclo directo del parásito, siendo un método de prevención cronometrado estratégicamente para impedirlo (Hoai, 2019). En el caso de la jaula colocada en estanques, si bien se aplica un manejo sanitario (encalado), el espejo de agua es menor y la concentración de materia orgánica favorece la replicación del parásito (De Torres y Meléndez, 1989).

Las parasitosis no llegaron a grados de moderado o severo que pudiera evidenciar un compromiso mayor de los alevinos evaluados, posiblemente debido a las condiciones de manejo de ambos tipos de crianza, ya que los parámetros físicoquímicos del agua, en ambos medios de crianza, no sobrepasaron los parámetros permisibles indicados por 
Cuadro 1. Parámetros de calidad de agua en el medio artificial y seminatural utilizado para la crianza de alevinos de gamitana (Colossoma macropomum) al inicio del experimento

\begin{tabular}{|c|c|c|c|c|c|}
\hline Calidad & Parámetro & $\begin{array}{l}\text { Medio } \\
\text { artificial }\end{array}$ & $\begin{array}{l}\text { Medio semi- } \\
\text { natural }\end{array}$ & $\begin{array}{c}\text { Límite } \\
\text { máximo } \\
\text { permisible }\end{array}$ & Referencia \\
\hline \multirow[t]{4}{*}{ Microbiológica } & $\begin{array}{c}\text { Coliformes } \\
\text { totales } \\
(\mathrm{NMP} / 100 \mathrm{ml})\end{array}$ & $16(5.9-33)$ & $23(8.1-53)$ & $<70$ & 2 \\
\hline & $\begin{array}{c}\text { Coliformes } \\
\text { termotolerantes } \\
(\mathrm{NMP} / 100 \mathrm{ml})\end{array}$ & $5.1(1.6-13)$ & $2.2(0.37-8.1)$ & $<14$ & 2 \\
\hline & $\begin{array}{c}\text { E. coli } \\
\text { (NMP/100 mL) }\end{array}$ & Ausente & Ausente & Ausente & 1.3 \\
\hline & $\begin{array}{l}\text { Pseudomona } \mathrm{sp} \\
(\mathrm{NMP} / 100 \mathrm{ml})\end{array}$ & Ausente & Ausente & Ausente & 1.3 \\
\hline \multirow[t]{8}{*}{ Fisicoquímica } & $\mathrm{pH}$ & 6.34 & 6.93 & $6.5-8.5$ & 1 \\
\hline & $\begin{array}{l}\text { Conductividad } \\
(\mu \mathrm{S} / \mathrm{cm})\end{array}$ & 48.5 & 44.1 & 1500 & 2 \\
\hline & $\begin{array}{l}\text { Turbiedad } \\
\text { (UNT) }\end{array}$ & 10.84 & 10.54 & 5 & 1 \\
\hline & $\begin{array}{c}\text { Dureza } \\
\mathrm{mg} \mathrm{CaCO}_{3} / 1\end{array}$ & 10 & 14 & 500 & 2 \\
\hline & $\begin{array}{c}\text { Hierro } \\
\mathrm{mg} \mathrm{Fe}^{++} / 1\end{array}$ & 0.5 & 0.45 & 0.30 & 2 \\
\hline & $\begin{array}{l}\text { Sulfatos } \\
\mathrm{mg} \mathrm{SO}_{4}^{-2} / 1\end{array}$ & 1.32 & 1.81 & 250 & 2 \\
\hline & $\begin{array}{l}\text { Cloruros } \\
\mathrm{Mg} \mathrm{Cl}^{-} / 1\end{array}$ & 5.13 & 5.99 & 250 & 2 \\
\hline & $\begin{array}{c}\text { Amonio } \\
\mathrm{mg} \mathrm{NH}_{3} / 1\end{array}$ & 0.11 & 0.10 & 1.5 & $*$ \\
\hline
\end{tabular}

1. Organización Mundial de la Salud (2004); 2. Estándares Nacionales de Calidad Ambiental para Agua (Decreto Supremo Nº02-2008-MINAM); 3. Resolución Ministerial 591-2008MINSA "Norma Sanitaria que establece los criterios microbiológicos de calidad sanitaria e inocuidad para los alimentos y bebidas de consumo humano"

* Las concentraciones de amonio adecuados para piscicultura deben ser menores a $1.5 \mathrm{mg} / \mathrm{l}$ UNT: Unidad Nefelométrica de turbiedad

la OMS (2004). En un estudio en peces Oreochromis niloticus que habitan el agua del río Nilo, se determinó que la contaminación con aguas residuales actuó como un fac- tor de estrés disminuyendo su capacidad inmune, permitiendo la entrada de protozoos externos como Trichodinas sp y Ambiphyra sp (Tayel et al., 2020). 
Cuadro 2. Grado de infestación por monogeneos Dactilogyrus spp en branquias de alevinos de gamitana en dos medios acuáticos de cultivo (Ucayali, Perú)

\begin{tabular}{ccc}
\hline Día & \multicolumn{2}{c}{ Grado de infección } \\
\cline { 2 - 3 } & $\begin{array}{c}\text { Medio } \\
\text { artificial }\end{array}$ & $\begin{array}{c}\text { Medio semi- } \\
\text { natural }\end{array}$ \\
\hline 1(inicio) & Negativo & Negativo \\
15 & Negativo & Leve (1) \\
30 & Negativo & Leve (1) \\
45 & Leve (1) & Negativo \\
60 & Leve (2) & Negativo \\
90 & Leve (2) & Leve (1) \\
\hline
\end{tabular}

\section{ConClusiones}

- No se observó diferencia significativa en el grado de parasitosis por monogeneos en branquias de alevinos de gamitana (Colossoma macropomum) entre los medios de crianza artificial y seminatural.

- Los niveles de parasitismo encontrados no representan un riesgo para infecciones moderadas o severas por monogeneos en branquias que afecte a las poblaciones de alevinos de gamitana.

\section{Literatura Citada}

1. Aly S, Fathi M, Youssef EM, Mabrok, M. 2020. Trichodinids and monogeneans infestation among nile tilapia hatcheries in Egypt: Prevalence, therapeutic and prophylactic treatments. Aquaculture Int 28: 1459-1471. doi:10.1007/s10499-02000537-w

2. Amanajás R, Silva J, Val A. 2018. Growing in the Dark Warmth: The case of Amazonian fish Colossoma macro- pomum. Front Mar Sci 5: 492. doi: 10.3389/fmars.2018.00492

3. Aragort W, León E, Guillén A, Silva, $M$, Balestrini C. 1997. Fauna parasitaria en peces de aguas continentales de interés económico para Venezuela. En: Memorias IV Encuentro Nacional de Acuicultura. Universidad Rómulo Gallegos.

4. Bakke TA, Cable J, Harris PD.2007. The biology of gyrodactylid monogeneans: the «Russian-doll killers. Adv Parasitol 64: 161-376. doi: 10.1016/ S0065-308X(06)-64003-7

5. Belmont-Jégu E, Domingues M, Martins M. 2004. Notozothecium Janauac-hensis n. sp. (Monogenoidea: Dacty-logyridae) from wild and cultured Tambaqui, Colossoma macropomum (Teleostei: Characidae: Serrasalminae) in Brazil. Zootaxa 1-8. doi: 10.5281/ zenodo. 158488

6. Bowers JM, Mustafa A, Speare DJ, Conboy GA, Brimacombe $M$, Sims DE, Burka JF. 2000. The physiological response of Atlantic salmon, Salmo salar $\mathrm{L}$ to a single experimental challenge with sea lice Lepeophtheirus salmonis. J Fish Dis 23: 165-172. doi: 10.1046/ j.1365-2761.2000.00225.x

7. Cohen SC, Justo MCN, Kohn A. 2013. South American monogenoidea parasites of fishes, amphibians and reptiles. Rio de Janeiro: Conselho Nacional de Desenvolvimento Científico e Tecnológico - CNPq. 664 p.

8. Collins MR, Rogers SG, Smith TIJ, Moser ML. 2000. Primary factors affecting sturgeon populations in the Southeastern United States: fishing mortality and degradation of essential habitats. Bull Marine Scie 66: 917-928.

9. Conroy $\boldsymbol{G}$ 2006. Principales enfermedades en peces de cultivo de la Amazo-nía. Acuicultura para la seguridad alimen-taria en comunidades aguarunas del alto Marañón, Perú. Procedimientos básicos para el reconocimiento y diagnóstico de las enfermedades y parásitos de los peces / Enfermedades parasitarias. Proyecto FAO: TCP/PER/3002 (A). p: 9-16 / 27-41. 56p. 
10. De Torres M, Melendez J. 1989. Pisciculturas en jaulas flotantes. Madrid: Ministerio de Agricultura, Pesca y Alimentación. $24 \mathrm{p}$.

11. Dinis N. 2014. Índices parasitarios en larvas, post larvas y alevinos de Piaractus brachypomus «paco» en relación a los factores ambientales, en el centro de investigaciones Quistococha del Instituto de Investigaciones de la Amazonia Peruana, Iquitos - Perú. Tesis de Maestría. Iquitos, Perú: Univ. Nacional de la Amazonia Peruana. $52 \mathrm{p}$.

12. Eiras J, Takeoto RM, Pavanelli GC. 2006. Métodos de estudo e técnicas laboratorais em parasitologia de peixes. Brasil: Univ. Estadual de Maringá. 199 p.

13. Flores J, Flores R. 2003. Monogeneos, parásitos de peces en México: estudio recapitulativo. Téc Pecu Méx 41: 175-192.

14. Fujimoto RY, Hide DMV, Paixão PEG, Abe HA, Dias JAR, Sousa NC, Maciel PO. 2019. Parasitic fauna and parasite-host relationship of tambaqui reared in São Francisco river basin, Brazilian northeast. Arq Bra Med Vet Zootec 71: 563-570. doi:10.1590/16784162-10306

15. Gomes LC, Chagas EC, Martins-Junior H, Roubach R, Ono EA, Lorenço JN. 2006. Cage culture of tambaqui (Colosssoma macropomum) in a central Amazon floodplain lake. Aquaculture 253: 374-384.

16. Gonzales JG. 2012. Parasitofauna en tilapia causante de mortalidad en alevinos en dos centros de cultivos, Lima, Perú. Neotrop Helminthol 6: 219-229.

17. Hoai TD. 2019. Reproductive strategies of parasitic flatworms (Platyhelminthes, Monogenea): the impact on parasite management in aquaculture. Aquaculture Int 28: 421-447.

18. Kingler RE, Floyd FR. 2002. Introduction to freshwater fish parasites. CIR716. Extension University of Florida. IFAS Extension. 13 p.

19. Kritsky DC, Thatcher VE, Kayton RJ. 1979. Neotropical Monogenoidea. 2. The Anacanthorinae Price, 1967, with the proposal of four new species of Anacanthorus Mizelle and Price, 1965, from Amazonian fishes. Acta Amaz 9: 355-361. doi: 10.1590/1809-43921979092355

20. Luo Y, Yang T. 2010. Seasonal population dynamics of the monogeneans Pseudorhabdosynochus coioidesis and $P$. serrani on wild versus cultured groupers in Daya Bay, South China Sea. Helminthologia 47: 241-250.

21. Martins L, Romero R. 1996. Efectos del parasitismo sobre el tejido branquial en peces cultivados: estudio parasitológico e histopatológico. Revta Bras Zool 13: 489-500.

22. [MINAM] Ministerio del Ambiente. 2008. Decreto Supremo N. ${ }^{\circ} 002-2008-$ MINAM, de 31 de julio, por el que se aprueban los estándares nacionales de calidad ambiental para agua. Diario Oficial El Peruano No 10311, 31/7/2008.

23. [MINSA] Ministerio de Salud. 2008. Resolución Ministerial N. ${ }^{\circ}$ 591-2008/ MINSA, de 27 de agosto, por el que se aprueba la Norma Sanitaria que establece los criterios microbiológicos de calidad sanitaria e inocuidad para los alimentos y bebidas de consumo humano. Diario Oficial El Peruano $N^{\circ} 10340,27 / 8 /$ 2008.

24. Murray AG, Peeler EJ. 2005. A framework for understanding the potential for emerging diseases in aquaculture. Prev Vet Med 67: 223235. doi: 10.1016/j.prevetmed.2004.10.012

25. [OMS] Organización Mundial de la Salud. 2004. Guías para la calidad del agua potable. Ginebra: OMS. 101 p.

26. Pontes AC. 1977. O pirarucú Arapaima gigas, (CUVIER, 1829), nos acudes publicos do Nordeste Brasileiro. Tesis de Maestría. Ceará, Brasil: Univ. Federal do Ceará. $50 \mathrm{p}$.

27. Scholz T, Kuchta R. 2005. Parásitos metazoarios de peces nativos y de cultivo en Amazonía, Perú. Tercer curso teórico y práctico sobre ictioparasitología. Instituto de parasitología, Acad. Cs. Rep. Checa. Iquitos Perú. 11-15 abril 2005. p: 1-9. 
28. Sierra CA, Maroso JJ. 2019. Estrategias de manejo para mejorar la producción piscícola en un sistema de jaulas flotantes con Tilapia roja (Oreochromis sp), en Montería, Córdoba. [Internet]. Disponible en: https://ciencia.lasalle.edu.co/ zootecnia/462

29. [SMEWW-APHA - AWWA -WEF] American Public Health Association American Water Works Association Water Environment Federation. 2012. Standard methods for examination of water and wastewater. $22^{\text {nd }}$ ed. Washington: American Public Health Association. $1360 \mathrm{p}$.

30. Sures B, Nachev M, Selbach C, Gliese DJ. 2017. Parasite responses to pollution: what we know and where we go in 'environmental parasitology'. Parasit Vectors 10: 65-65.

31. Tayel I, Ahmed M, Ramadan A. 2020. Histopahological alterations and parasitic infection in Oreochromis niloticus fish inhabiting the River Nile water. Egyp J
Acuatic Biol Fish 24: 233-247. doi: 10.21608/ejabf.2020.70036

32. Thatcher VE, Kritsky DC. 1983. Neotropical Monogenoidea. 4. Linguadactyloides brinkmanni gen. et sp. n. (Dactylogyridae: Linguadactyloidinae subfam. nov.) with observations on the pathology in a Brazilian freshwater fish, Colossoma macropomum (Cuvier). Proc Helminthol Soc Washington 50: 305-331.

33. Vargas M, Sandoval $N$, Casas E, Pizango G, Manchego A. 2015. Parásitos y lesiones histopatológicas en branquias de gamitanas (Colossoma macropomum) juveniles bajo crianza semiintensiva. Rev Inv Vet Perú 26: 577 586. doi: 10.15381/rivep.v26i4.11222

34. Winger AC, Kanck M, Kristoffersen R, Knudsen R. 2008. Seasonal dynamics and persistence of Gyrodactylus salaris in two riverine anadromous Arctic charr populations. Environ Biol Fish 83: 117-123. 